\title{
Prevalence of 25 canine behavioral problems and relevant factors of each behavior in Japan
}

\author{
Ryoko YAMADA ${ }^{1)}$, Sayaka KUZE-ARATA ${ }^{1,2)}$, Yasushi KIYOKAWA ${ }^{1)}$ and \\ Yukari TAKEUCHI ${ }^{1) *}$ \\ 1)Laboratory of Veterinary Ethology, Graduate School of Agricultural and Life Sciences, \\ The University of Tokyo, Yayoi 1-1-1, Bunkyo-ku, Tokyo 113-8657, Japan \\ 2) Present address: Department of Animal Science and Biotechnology, Azabu University, \\ Fuchinobe 1-17-71, Chuo-ku, Sagamihara, Kanagawa 252-5201, Japan
}

J. Vet. Med. Sci.

81(8): 1090-1096, 2019

doi: 10.1292/jvms.18-0705

Received: 28 November 2018 Accepted: 23 May 2019 Advanced Epub: 5 June 2019

\begin{abstract}
Undesirable behaviors exhibited by dogs are called canine behavioral problems. Behavioral problems are common among dogs kept in ordinary homes; hence, veterinarians need to have sufficient knowledge of these problems, so as to advise owners. Among dogs kept in ordinary Japanese homes, only a few behaviors have been investigated regarding prevalence and relevant factors. To improve understanding of behavioral problems, a comprehensive survey is crucial. In this study, we distributed an online survey including general information and questions about 25 canine behaviors that can become problematic to owners, to investigate (1) the prevalence of behavioral problem, (2) the most common behavioral problems, and (3) the relevant factors of each behavior. We collected 2,050 valid responses. Among all dogs, $86.0 \%$ exhibited at least one behavioral problem. "Barking at noises inside the house", "Barking at unfamiliar visitors", and "Pica" were the most frequent behavioral problems. To reveal those factors related to the expression of each behavior, we performed multiple logistic regression analyses, with the presence of behaviors as objective variables and dogs' general information as explanatory variables. Breed, age, the presence of other dogs in the household, sex, neutering status, source of acquisition, and type of residence were associated with 19, 16, 5, 4, 4, 4, and 1 behaviors, respectively. This study revealed the prevalence of 25 canine behavioral problems in Japan and relevant factors of each behavior. Our findings will be useful for veterinarians in advising owners about preventing behavioral problems.
\end{abstract}

KEY WORDS: behavioral problem, canine, online questionnaire survey, prevalence, relevant factor

A "behavioral problem" refers to behavior exhibited by an animal that is unacceptable to the owner, regardless of its level of abnormality $[1,6]$. Therefore, exactly the same behavior can be regarded as either a behavioral problem or non-problematic normal behavior, depending on the owner's feelings. Despite such ambiguity, behavioral problems are becoming a common concern among dog owners. It has been reported that more than $80 \%$ of dogs kept in homes exhibit behavioral problems [9, 22, 35].

It is known that common behavioral problems vary among different countries [9, 22, 23, 35]. A survey conducted in Australia reported that the main behavioral problems were overexcitement, jumping up on people, and rushing at people or dogs [23]. In South Korea, excessive barking, inappropriate elimination, and aggressive behavior are the most frequent behavioral problems [9]. Even within the same country, common behavioral problems can vary according to region. For example, in Iran, jumping up, aggression toward dogs, and aggression toward people are reported as the most frequent behavioral problems in Tehran [35] whereas excessive activity, inappropriate elimination, and fearfulness are the main behavioral problems in Mashhad [22]. Given that dog owners usually consult veterinarians regarding behavioral problems $[15,38]$, it is important for veterinarians, especially general practitioners, to have good knowledge about the most frequent behavioral problems in their area.

To control behavioral problems, veterinarians are also expected to advise owners regarding how to prevent the onset of these problems in their dog. It was reported that the dog's appearance is a most important reason for choosing a dog [39]. Such owners can become annoyed by their dog's unexpected behavioral characteristics. Moreover, once behavioral problems progress, it is difficult to cure them. For example, $48.8 \%, 32.5 \%$, and $32.1 \%$ of dogs showing aggression toward their owners, aggression toward

*Correspondence to: Takeuchi, Y.: aytake@mail.ecc.u-tokyo.ac.jp

(Supplementary material: refer to PMC https://www.ncbi.nlm.nih.gov/pmc/journals/2350/)

(c2019 The Japanese Society of Veterinary Science

This is an open-access article distributed under the terms of the Creative Commons Attribution Non-Commercial No Derivatives (by-nc-nd) License. (CC-BY-NC-ND 4.0: https://creativecommons.org/licenses/by-nc-nd/4.0/) 
strangers, and separation anxiety, respectively, did not improve with behavioral treatment [34]. Given that any behavior can become a behavioral problem once the behavior appears, veterinarians are recommended to understand the factors related to the presence of a wide variety of behaviors, so as to properly advise any owner. Although several studies targeting particular breeds have revealed the relevant factors in one or several behaviors $[2,3,8,11,12,14,16,17,34]$, to the best of our knowledge, comprehensive analyses are still lacking.

In Japan, little attention has been paid to canine behavioral problem. Indeed, clinical behavioral medicine was included in the curriculum for the veterinary license in 2011 [37]. As a consequence, the available information for Japan is still limited. One survey of $100 \mathrm{dogs}$ that visited a veterinary clinic for reasons other than behavioral problems found that $82.0 \%$ of dogs exhibited behavioral problems, most commonly destructive behavior, barking, and aggression toward people [36]. Another survey focusing on anxiety-related behaviors at pet shops in three large cities in Japan found that approximately 20\% of 262 dogs troubled their owner with the behaviors [24]. To achieve a better understanding of behavioral problems in Japanese homes, a comprehensive survey of behavioral problems and analyses of the factors related to such behaviors are crucial.

In the present study, we conducted an online survey to obtain information regarding 25 canine behaviors. We queried the frequency and degree of owner annoyance for each behavior. When the expressed behavior bothered the owner, we regarded the behavior as a behavioral problem, regardless of the frequency, abnormality or degree of annoyance. We then analyzed (1) the prevalence of behavioral problem and (2) the most common behavioral problems. In addition, we analyzed (3) the relevant factors for the expression of each behavior, to improve understanding of these 25 behaviors.

\section{MATERIALS AND METHODS}

\section{Online questionnaire}

We developed an original online questionnaire based on the C-BARQ [20] and previous studies [3, 4, 9, 11, 22, 23, 35, 36], as well as based on complaints by dog owners at the Veterinary Behavior Clinic in the Veterinary Medical Center of The University of Tokyo. On the questionnaire, we queried general information of owners and dogs, and the frequency and the degree of annoyance for 25 behaviors. General information included the owner's age and sex; the residential area and type of residence; the number of dogs in the household; the dog's breed, age, body weight, sex, neutering status, age at neutering, living area, source of acquisition, and age at acquisition (Table S1).

Regarding dog behaviors, each behavior was described in detail on the questionnaire, as shown in Table S1. Respondents were asked to report the frequency of each behavior within the last 3 months, using a 5 -point scale ( $5=$ always, $4=$ usually, $3=$ sometimes, $2=$ rarely, $1=$ never). If respondents were unsure or could not rate the behavior using this scale, they were asked to answer NA (unknown or not applicable). Respondents were also asked to report the degree to which they were troubled by these behaviors, using a 4-point scale (4=very troubled, $3=$ moderately troubled, $2=$ not really troubled or $1=$ never troubled).

\section{Respondents and data collection}

Voluntary respondents were recruited in February 2018 from among subscribers of an online magazine published by the online pet products store "Doctor's Advice PEPPY" (Shinnippon Calendar, Osaka, Japan). Responses were collected without any limitation regarding dogs' status, for example, breed or age. Respondents with multiple dogs were requested to answer the survey questions for only one specific dog. After omitting participants' personal information, such as name and e-mail address, all responses were given to the authors. The following responses were excluded from analyses; responses lacking any general information, responses with more than two answers of NA, and responses regarding any behavior for which a respondent reported being moderately troubled (rating of 3) or very troubled (rating of 4) but reported a behavior frequency of "never" (rating of 1). In cases of duplicated responses, one duplicate was excluded. This survey was conducted with the approval of the Ethics Review Committee of the Faculty of Agriculture at The University of Tokyo.

\section{Data analyses}

When respondents reported that their dog exhibited a particular behavior (ratings from 2 to 5) and that they were troubled by the behavior (ratings from 3 to 4), the behavior was considered a behavioral problem. We calculated the percentage of dogs with at least one behavioral problem, to determine the prevalence of behavioral problem. We also calculated the number of dogs exhibiting each behavior, to reveal the most common behavioral problems.

Given that any behavior can become a behavioral problem once the behavior appears, we investigated factors related to the expression of each behavior in the present study. Because the Shapiro-Wilk test revealed that the frequency of behavior did not follow a normal distribution, we converted the frequency of behavior into the presence (ratings of 2 and more) or absence (rating of 1) of behavior, used as objective variables in multiple logistic regression analyses. Responses of NA were excluded from the multiple logistic regression analysis. General information used as explanatory variables included the following: breed (categories: Shiba Inu, Toy Poodle, Miniature Dachshund, Chihuahua, or other breeds), age (categories: young ( $<2$ years old), adult $(2-6$ years old), senior (7-10 years old), or geriatric ( $\geq 11$ years old)), sex (categories: female or male), neutering status (categories: intact or neutered), source of acquisition (categories: breeder, pet store, rescue, or other sources), type of residence (categories: house or apartment), and presence of other dogs in the household (categories: no or yes). We chose four breeds as the independent categories of breed because there were surveys completed for more than 100 dogs for each of these four breeds, which seemed sufficient for the analyses. Other dogs were merged into the "other breeds" category. Details of the other breeds are shown in Table S2. Age was 
categorized as above, to assess differences in the odds of each behavior among age groups. As for the source of acquisition, type of residence, and presence of other dogs in the household, some answers were merged into one category (Table S1). Other breeds and adult dogs were treated as the reference for breed and age. For the other factors, the first category listed above for each factor was treated as the reference. We did not include owner's age and sex as explanatory variables because we focused on factors associated with the dog, which is more useful to veterinarians. Residential area was not included as an explanatory variable because this information was subjective. In addition, we did not include information of the dog's body weight, age at neutering, living area, and age at acquisition; instead, we included information regarding breed; we used neutering status as an explanatory variable regarding neutering; most dogs lived inside the home; and the age at acquisition was related to the source of acquisition, respectively.

Based on Bonferroni correction ( $0.05 / 25$ behaviors), significance was set at $P<0.002$. Odds ratios calculated in multiple logistic regression analyses were presented with $99.8 \%$ confidence intervals. All analyses were conducted using R version 3.4.4 [30].

\section{RESULTS}

\section{Basic information}

Of 2,359 responses on the questionnaire, 2,050 were valid. Responses were collected for 78 purebred dogs and mixed breed dogs (Table S2). Shiba Inu ( $n=297,14.5 \%)$, Toy Poodle $(n=250,12.2 \%)$, Miniature Dachshund $(n=177,8.6 \%)$, and Chihuahua $(n=142$, $6.9 \%)$ were the four most common dog breeds. Regarding age, dogs were classified as young $(n=198,9.7 \%)$, adult $(n=795,38.8 \%)$, senior $(n=575,28.0 \%)$, and geriatric $(n=482,23.5 \%)$. There were $1,006(49.1 \%)$ female dogs (intact $n=180$, neutered $n=826)$ and $1,044(50.9 \%)$ male dogs (intact $n=294$, neutered $n=750)$. Respondents obtained their dogs from a pet store $(n=1,035,50.5 \%)$, breeder $(n=563,27.5 \%)$, rescue $(n=211,10.3 \%)$, and other sources $(n=241,11.8 \%)$. Regarding type of residence, $1,519(74.1 \%)$ and $531(25.9 \%)$ dogs lived in a house and an apartment, respectively. A total of 1,498 (73.1\%) dogs were the only dog in the household.

As for dog owners, 70 (3.4\%) were $\leq 29$ years old, 1,698 (82.8\%) were aged 30-59 years, and $282(13.8 \%)$ were aged $\geq 60$ years. The number of female and male respondents was $1,832(89.4 \%)$ and $218(10.6 \%)$, respectively. A total 562 (27.4\%), 907, (44.2\%), and $581(28.3 \%)$ respondents lived in urban, suburban, and rural areas, respectively.

\section{Prevalence of each behavioral problem}

Response rates to questions about the frequency of behavior ranged from 97.0 to $99.8 \%$. As a whole, 1,764 (86.0\%) respondents stated that they were moderately or very troubled (ratings of 3 or 4 ) by at least one behavior in their dog.

Figure 1 shows the number of dogs whose behavior bothered their owner and the number who exhibited each behavior. All 25 behaviors could be regarded as behavioral problems. The most three common behaviors that troubled respondents were "Barking at noises inside the house $(n=843,41.1 \%$ of 2,050 dogs)", "Barking at unfamiliar visitors" ( $n=787,38.4 \%)$, and "Pica" ( $n=715$, $34.9 \%)$.

\section{Relevant factors of each behavior}

The number of dogs included in each multiple logistic regression analysis was between 1,988 and 2,046. McFadden's pseudo R-squared in each analysis ranged from 0.01 to 0.12 (Table S3).

The general information of dogs was significantly associated with 22 behaviors, except "Fear of loud noises, such as thunder and/or fireworks", "Staring or snapping at the air", and "Staring at or chasing lights or shadows". Dog breed, age, the presence of other dogs in the household, sex, neutering status, source of acquisition, and type of residence were associated with 19, 16, 5, 4, 4, 4, and 1 behaviors, respectively (Table 1 and Table S3). As for the most three common behavioral problems, the odds of "Barking at noises inside the house" were significantly higher in Toy Poodles and Miniature Dachshunds but significantly lower in Shiba Inus than in other breeds. Moreover, the odds of this behavior were significantly lower in young dogs and geriatric dogs than in adult dogs. The odds of "Barking at unfamiliar visitors" were significantly higher in Toy Poodles, Miniature Dachshunds, and Chihuahuas than in other breeds. Moreover, the odds of this behavior were significantly lower in young dogs and geriatric dogs than in adult dogs, and it was also significantly lower among dogs living in an apartment than among dogs living in a house. The odds of "Pica" were significantly higher in young dogs and significantly lower in senior and geriatric dogs. In addition, the odds of this behavior was significantly higher among neutered dogs than intact dogs (Table 1).

\section{DISCUSSION}

In this study, we found that $86.0 \%$ of dogs exhibited at least one behavioral problem. These results suggest that canine behavioral problems in Japan are as common as in the other countries. For example, the prevalence of behavioral problems has been reported as 84.5, 85.6, and 94.9\% in South Korea, and in Mashhad and Teheran in Iran [9, 22, 35]. Because the number of behaviors investigated in our study ( 25 behaviors) was the largest, we expected that the prevalence would be highest in our study. Theoretically, the greater the number of included behaviors, the higher the percentage of respondents who report being troubled by at least one behavior. However, the prevalence was almost the same or slightly lower than that of previous studies. One reason for this might be that prevalence has been calculated based on the frequency of behavior in previous studies [9, 22, 35]. Therefore, it is possible that some respondents are not annoyed even if their dog exhibits the investigated behaviors. In any case, there is still a great demand for the treatment of behavioral problems among dogs kept in Japanese ordinary homes. 


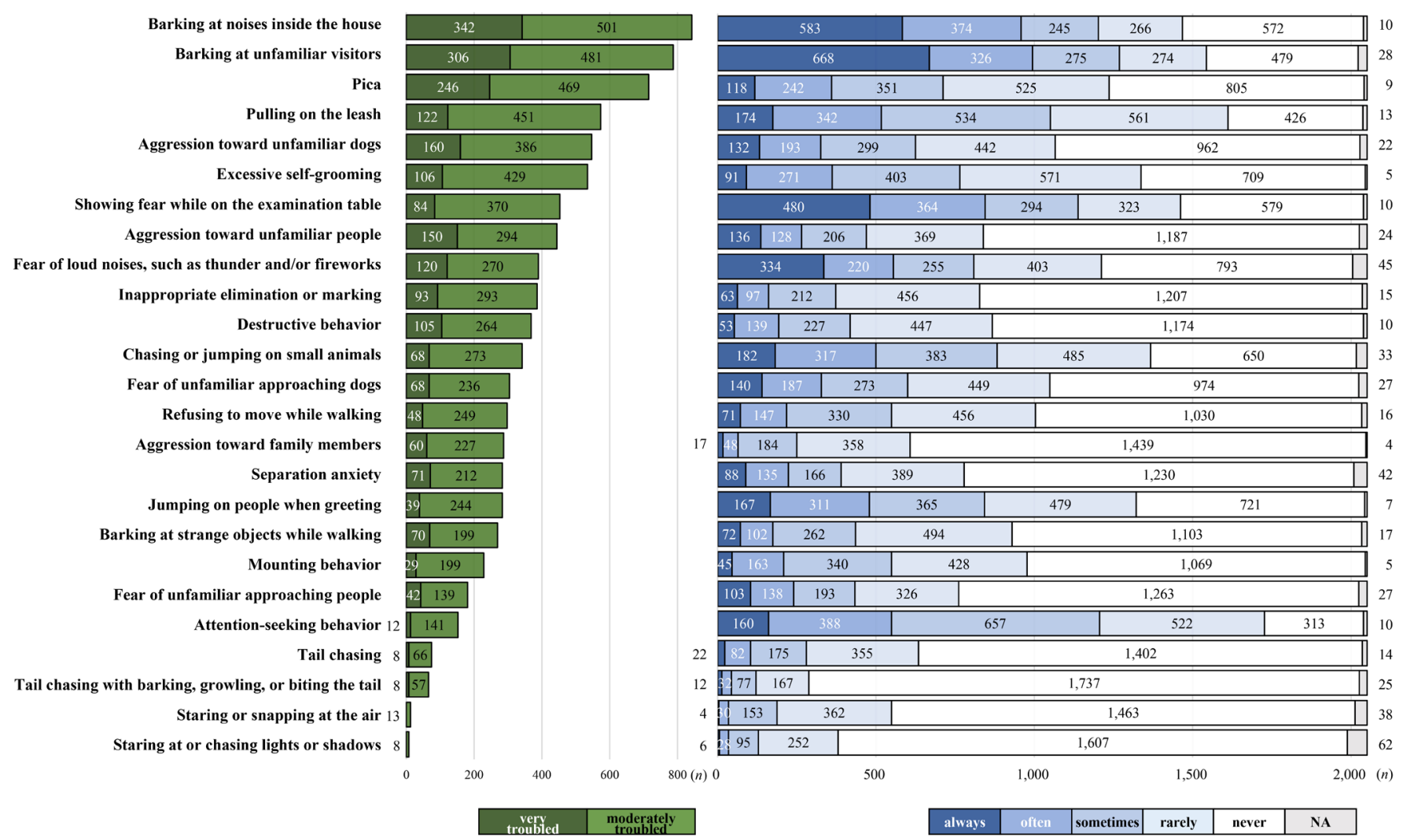

Fig. 1. The number of dogs troubling their owner and exhibiting each behavior. The left panel shows the number of dogs whose owner reported that their dog exhibited the behavior (from "always" to "rarely") and "very" or "moderately" troubled them. The right panel shows the number of dogs exhibiting each behavior. The total number of dogs is 2,050 .

In the present study, we found that "Barking at noises inside the house" and "Barking at unfamiliar visitors" were the first and second most common behavioral problems. One interpretation could be that breed preference and distress caused by barking led to these behaviors becoming the most common behavioral problems. Toy Poodles, Chihuahuas, and Miniature Dachshunds were the main breeds in this study, which is consistent with popular breeds in Japan [21]. These three breeds presented significantly higher odds of "Barking at unfamiliar visitors" than other breeds. In addition, Toy Poodles and Miniature Dachshunds showed significantly higher odds of "Barking at noises inside the house" than other breeds. Moreover, other popular breeds in Japan [21] such as Kaninchen Dachshund, Maltese, Pomeranian, Yorkshire Terrier, and Beagle were also reported that they frequently exhibited excessive barking [33]. Given that most of the popular breeds in Japan have a predisposition for barking, it is natural that these behaviors are highly prevalent in Japanese homes. In addition, these behaviors showed not only a high prevalence rate but also a high percentage of respondents who were troubled by the behaviors. This might be because barking causes distress or disrupts the life of owners, family members, and neighborhoods. It was reported that the bark of a dog can reach $90 \mathrm{~dB}$ [28], which exceeds the reference noise level $(55 \mathrm{~dB})$ [27] for residential areas in Japan. Taken together, the interaction between breed preference and distress from barking rendered these behaviors common behavioral problems.

In this study, we found that the odds of "Barking at noises inside the house" and "Barking at unfamiliar visitors" were significantly lower in young dogs than adult dogs. Given that many respondents were troubled by these behaviors, this result suggests that even though a dog does not exhibit these behaviors while it is young, it might increasingly exhibit these behaviors as it grows, and consequently owners might become irritated by them once the dog becomes an adult. It has been reported that dogs bark for various reasons such as fear, anxiety, pain, territorial defense, threatening, greeting, play solicitation, contact seeking, and social facilitation $[5,10,19]$. Although we did not investigate the motivation for barking in this study, fear of particular stimuli might be related to both "Barking at noises inside the house" and "Barking at unfamiliar visitors", according to the target of barking in the survey questions. Previous studies have indicated that puppies that had been exposed to various noises or people were less likely to later show fear responses to these $[13,25]$. Therefore, familiarizing a dog with such stimuli early in life might result prevent these behaviors when the dog is grown. When owners take their puppy to a veterinary clinic for vaccination or a physical examination, veterinarians are strongly encouraged to recommend that their clients expose the dog to people and noises, even if owners report having no difficulties with their dog's behavior at that point. In addition, owners' inappropriate responses to their dog's barking might increase the behavior. If owners pay attention to their dog (including eye contact or saying "no") while or after it barks, this will usually result in making the barking response stronger [19]. To prevent barking from getting worse, it 


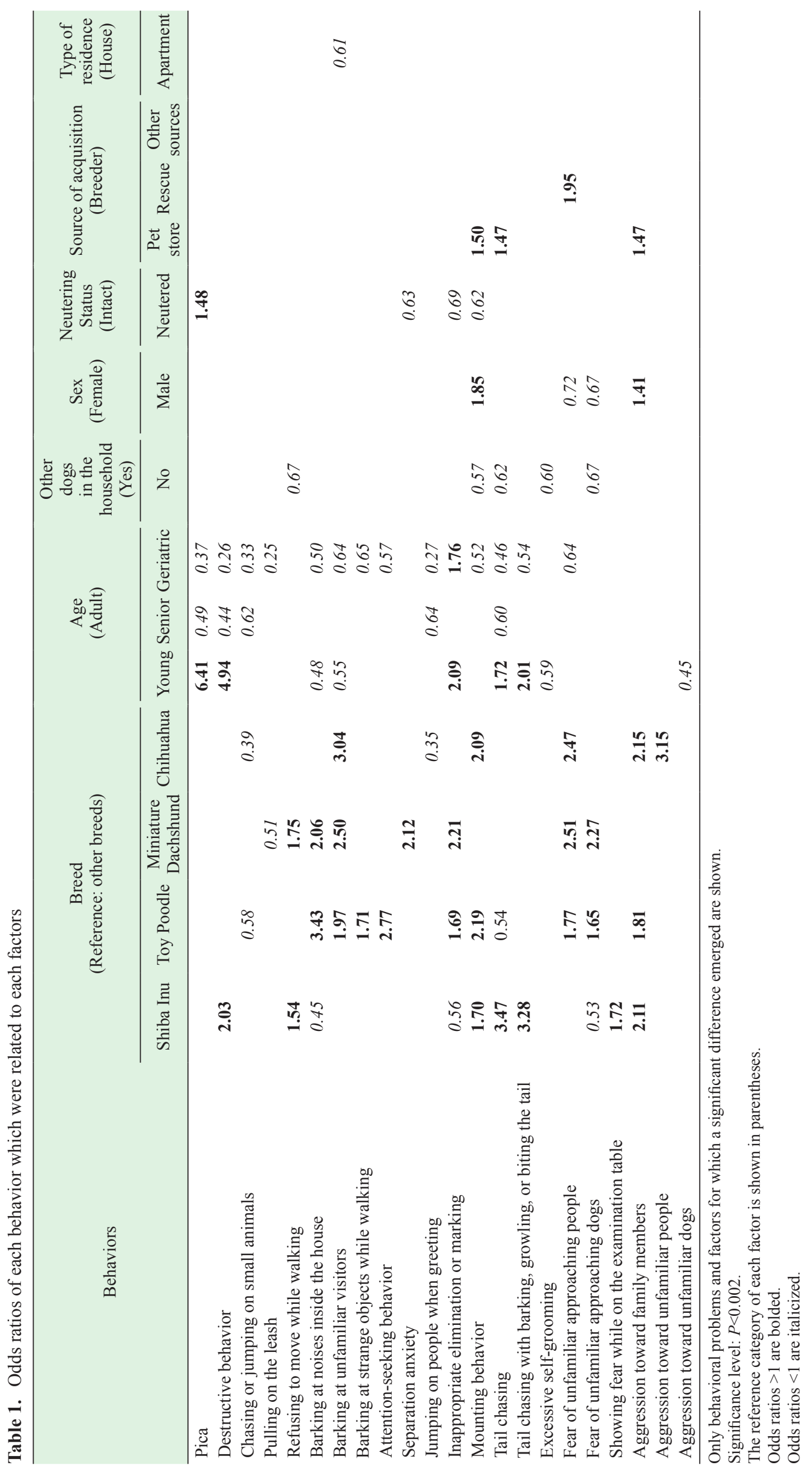


would be important to teach the dog how to settle down and relax while it is still young, and to pay no attention to barking. Thus, it is important for veterinarians to provide their clients with information concerning the prevention of and/or treatment for barking, especially owners of Toy Poodles, Miniature Dachshunds, and Chihuahuas.

"Pica" was the third more commonly reported behavioral problem in this study. We found that the odds of "Pica" were higher in young dogs than adult dogs, and higher in neutered dogs than intact dogs. These results suggest that owners of young dogs and neutered dogs need to be very careful to prevent this behavior. Because pica can impair a dog's health or even cause death [7, 18], many participants would be worried about this behavior. In the present study, young dogs showed higher odds of "Pica" than adult dogs. This result was similar to that of previous research that targeted dogs diagnosed with pica by a veterinarian; dogs aged less than 1 year, 1 year, and 2 years accounted for $4.4 \%, 2.5 \%$, and $1.8 \%$ of all cases of pica, whereas each 1 -year age group from 3 to 10 years accounted for under $1.5 \%$ of all cases [32]. The odds of "Pica" was significantly lower in adult dogs than in young dogs, and significantly lower in senior and geriatric dogs than adult dogs, that is, the prevalence of pica would dramatically decrease with age. Therefore, many owners of young dog who have been troubled by pica might be relieved from this problem once the dog grows up. In addition, we found that neutered dogs showed significantly higher odds of "Pica" than intact dogs. This might be because gonadectomy increases a dog's appetite [26, 29]. In any case, pica can be prevented by removing substances that are likely to be bitten off or swallowed by a dog from its living area. Veterinarians are strongly encouraged to recommend that owners of young dogs and neutered dogs provide a safe environment for their dog.

In the present study, we found the relevant factors of 25 included behaviors. To provide advice that is tailored to each owner, veterinarians can use our findings regarding the relationships between the investigated general information and behaviors (shown in Table 1 and Table S3). Among the factors analyzed, breed and age were related to more behaviors than other factors. As for breed, Shiba Inus, Toy Poodles, Miniature Dachshunds, and Chihuahuas exhibited higher odds for 7, 9, 7, and 5 behaviors than other breeds, respectively. Given that the behaviors investigated in the present study can become behavioral problems, these four breeds, which are among the most popular in Japan [21], might cause distress for their owners more often than other breeds. To prevent unexpected behavioral issues, it would be important to inform people about the behavioral characteristics of certain breeds before they acquire or adopt a dog so that they can choose a breed that conforms to their lifestyle or living environment. In addition, the present study revealed that the odds of the included behaviors changed markedly with age. This result suggests that owners need to pay attention to different behaviors according to their dog's age. However, although breed and age were the main relevant factors in the present study, the other factors are also important for veterinarians; these factors are related to serious behavioral problems such as aggression, anxiety-related behaviors, barking, and inappropriate elimination or marking. Indeed, these behaviors have been reported as reasons for taking dogs to veterinary behavioral clinics or for abandoning dogs [3, 11, 31]. Because any behavior can be a behavioral problem depending on the owner's feelings, veterinarians need to have broad knowledge regarding factors relevant to various behaviors that might be considered problematic.

It should be noted that there were some limitations in this study. First, the present results might be biased because respondents were subscribers of an online magazine published by an online pet products store. Second, the contents of the questionnaire were limited; details of each behavior were not investigated. In addition, the interpretation of the terms used to query the frequency and degree of annoyance might differ depending on respondents; however, this would have little effect on the present findings because the presence/absence of behaviors and owners feeling bothered/not bothered by behaviors would not be affected by different interpretations of the wording in the survey. Third, values for McFadden's pseudo R-squared of the logistic regression analyses were considered to be extremely low. This could be because other factors not included in this study, such as training and walking schedule are also related to the appearance of these behaviors [9, 35]. In addition, causal relationships between the relevant factors and behaviors were not investigated. A longitudinal study is needed to confirm behavioral changes according to age, neutering status, type of residence, and presence of other dogs in the household.

In conclusion, this study revealed the prevalence of 25 canine behavioral problems and relevant factors of each behavior in Japan. Among all dogs, $86.0 \%$ troubled their owner. "Barking at noises inside the house", "Barking at unfamiliar visitors" and "Pica" were the common behavioral problems. In addition, we revealed general information that were related to the expression of these 25 behaviors. The results of this study would be useful for veterinarians, who can then better advise their clients regarding how to prevent behavioral problems in their dogs.

CONFLICT OF INTEREST. The authors declare no conflicts of interest.

ACKNOWLEDGMENTS. We appreciate Mr. Ryoji Nishizawa for his cooperation in distributing the online questionnaire. This work was supported by grants from the Japan Society for the Promotion of Science Grants-in-Aid for Scientific Research (JSPS KAKENHI, Tokyo, Japan; grant number 26292163).

\section{REFERENCES}

1. Amat, M., de la Torre, J. L. R., Fatjó, J., Mariotti, V. M., Van Wijk, S. and Manteca, X. 2009. Potential risk factors associated with feline behaviour problems. Appl. Anim. Behav. Sci. 121: 134-139. [CrossRef]

2. Arata, S., Takeuchi, Y., Inoue, M. and Mori, Y. 2014. "Reactivity to stimuli" is a temperamental factor contributing to canine aggression. PLoS One 9: e100767. [Medline] [CrossRef]

3. Bamberger, M. and Houpt, K. A. 2006. Signalment factors, comorbidity, and trends in behavior diagnoses in dogs: 1,644 cases (1991-2001). J. Am. 
Vet. Med. Assoc. 229: 1591-1601. [Medline] [CrossRef]

4. Beaver, B. V. 1994. Owner complaints about canine behavior. J. Am. Vet. Med. Assoc. 204: 1953-1955. [Medline]

5. Blackwell, E. J., Bradshaw, J. W. S. and Casey, R. A. 2013. Fear responses to noises in domestic dogs: Prevalence, risk factors and co-occurrence with other fear related behaviour. Appl. Anim. Behav. Sci. 145: 15-25. [CrossRef]

6. Borchelt, P. L. and Voith, V. L. 1982. Classification of animal behavior problems. Vet. Clin. North Am. Small Anim. Pract. 12: 571-585. [Medline] [CrossRef]

7. Burton, A. G., Talbot, C. T. and Kent, M. S. 2017. Risk factors for death in dogs treated for esophageal foreign body obstruction: A retrospective cohort study of 222 cases (1998-2017). J. Vet. Intern. Med. 31: 1686-1690. [Medline] [CrossRef]

8. Casey, R. A., Loftus, B., Bolster, C., Richards, G. J. and Blackwell, E. J. 2014. Human directed aggression in domestic dogs (Canis familiaris): Occurrence in different contexts and risk factors. Appl. Anim. Behav. Sci. 152: 52-63. [CrossRef]

9. Chung, T.h., Park, C., Kwon, Y.m. and Yeon, S.c. 2016. Prevalence of canine behavior problems related to dog-human relationship in South Korea -A pilot study. J. Vet. Behav. 11: 26-30. [CrossRef]

10. Cohen, J. A. and Fox, M. W. 1976. Vocalizations in wild canids and possible effects of domestication. Behav. Processes 1: 77-92. [Medline] [CrossRef]

11. Col, R., Day, C. and Phillips, C. J. C. 2016. An epidemiological analysis of dog behavior problems presented to an Australian behavior clinic, with associated risk factors. J. Vet. Behav. 15: 1-11. [CrossRef]

12. Cross, N. J., Rosenthal, K. and Phillips, C. J. 2009. Risk factors for nuisance barking in dogs. Aust. Vet. J. 87: 402-408. [Medline] [CrossRef]

13. Cutler, J. H., Coe, J. B. and Niel, L. 2017. Puppy socialization practices of a sample of dog owners from across Canada and the United States. J. Am. Vet. Med. Assoc. 251: 1415-1423. [Medline] [CrossRef]

14. Duffy, D. L., Hsu, Y. and Serpell, J. A. 2008. Breed differences in canine aggression. Appl. Anim. Behav. Sci. 114: 441-460. [CrossRef]

15. Fatjó, J., Ruiz-de-la-Torre, J. L. and Manteca, X. 2006. The epidemiology of behavioural problems in dogs and cats: A survey of veterinary practitioners. Anim. Welf. 15: 179-185.

16. Flint, H. E., Coe, J. B., Serpell, J. A., Pearl, D. L. and Niel, L. 2017. Risk factors associated with stranger-directed aggression in domestic dogs. Appl. Anim. Behav. Sci. 197: 45-54. [CrossRef]

17. Goto, A., Arata, S., Kiyokawa, Y., Takeuchi, Y. and Mori, Y. 2012. Risk factors for canine tail chasing behaviour in Japan. Vet. J. 192: $445-448$. [Medline] [CrossRef]

18. Hayes, G. 2009. Gastrointestinal foreign bodies in dogs and cats: a retrospective study of 208 cases. J. Small Anim. Pract. 50: 576-583. [Medline] [CrossRef]

19. Horwitz, D. F. and Neilson, J. C. 2007. Vocalization: canine and feline. pp. 514-522. In: Blackwell's Five-minute Veterinary Consult Clinical Companion: Canine and Feline Behavior, 1st ed. (Horwitz, D. F. and Neilson, J. C. eds.), Blackwell Publishing, Ames.

20. Hsu, Y. and Serpell, J. A. 2003. Development and validation of a questionnaire for measuring behavior and temperament traits in pet dogs. J. Am. Vet. Med. Assoc. 223: 1293-1300. [Medline] [CrossRef]

21. Japan Kennel Club. https://www.jkc.or.jp/modules/publicdata/index.php?content_id=20 (in Japanese) [accessed on October 15, 2018].

22. Khoshnegah, J., Azizzadeh, M. and Mahmoodi Gharaie, A. 2011. Risk factors for the development of behavior problems in a population of Iranian domestic dogs: Results of a pilot survey. Appl. Anim. Behav. Sci. 131: 123-130. [CrossRef]

23. Kobelt, A. J., Hemsworth, P. H., Barnett, J. L. and Coleman, G. J. 2003. A survey of dog ownership in suburban Australia -conditions and behaviour problems. Appl. Anim. Behav. Sci. 82: 137-148. [CrossRef]

24. Kurachi, T., Irimajiri, M., Mizuta, Y. and Satoh, T. 2017. Dogs predisposed to anxiety disorders and related factors in Japan. Appl. Anim. Behav. Sci. 196: 69-75. [CrossRef]

25. Kutsumi, A., Nagasawa, M., Ohta, M. and Ohtani, N. 2013. Importance of puppy training for future behavior of the dog. J. Vet. Med. Sci. 75: 141-149. [Medline] [CrossRef]

26. Maarschalkerweerd, R. J., Endenburg, N., Kirpensteijn, J. and Knol, B. W. 1997. Influence of orchiectomy on canine behaviour. Vet. Rec. 140: 617-619. [Medline] [CrossRef]

27. Ministry of the Environment Government of Japan. https://www.env.go.jp/kijun/oto1-1.html (in Japanese) [accessed on Aplil 9, 2019].

28. Ministry of the Environment Government of Japan. https://www.env.go.jp/nature/dobutsu/aigo/2_data/arikata/h16_03/mat02.pdf (in Japanese) [accessed on Aplil 9, 2019].

29. O'Farrell, V. 1990. Behavioural effects of ovariohysterectomy on bitches. J. Small Anim. Pract. 31: 595-598. [CrossRef]

30. R Core Team. 2018. R: A language and environment for statistical computing. R Foundation for Statistical Computing, Austria. https://www.rproject.org/ [accessed on May 29, 2018].

31. Salman, M. D., Hutchison, J., Ruch-Gallie, R., Kogan, L., New, J. C., Kass, P. H. and Scarlett, J. M. 2000. Behavioral reasons for relinquishment of dogs and cats to 12 shelters. J. Appl. Anim. Welf. Sci. 3: 93-106. [CrossRef]

32. Shimamura, A., Inoue, M. and Arai, T. 2011. A research on foreign body ingestion in dogs. J. Vet. Epidemiol 16: 35-36. [CrossRef]

33. Takeuchi, Y. and Mori, Y. 2006. A comparison of the behavioral profiles of purebred dogs in Japan to profiles of those in the United States and the United Kingdom. J. Vet. Med. Sci. 68: 789-796. [Medline] [CrossRef]

34. Takeuchi, Y., Ogata, N., Houpt, K. A. and Scarlett, J. M. 2001. Differences in background and outcome of three behavior problems of dogs. Appl. Anim. Behav. Sci. 70: 297-308. [Medline] [CrossRef]

35. Tamimi, N., Malmasi, A., Talebi, A., Tamimi, F. and Amini, A. 2013. Owner complaints of canine behavior in Iran -A preliminary survey. J. Vet. Behav. 8: 26-31. [CrossRef]

36. Uchida, Y., Yamada, K., Nakade, T. and Otomo, K. 1996. Owner complaints about canine and feline behavior. Nippon Juishikai Zasshi 49: 337-341.

37. Veterinary Medicine Model Core Curriculum. https://www.jaeve.org/cur/release/ (in Japanese) [accessed on October 15, 2018].

38. Voith, V. L. 1984. Why should veterinarians study animal behavior? Mod. Vet. Pract. 65: 363-364. [Medline]

39. Weiss, E., Miller, K., Mohan-Gibbons, H. and Vela, C. 2012. Why did you choose this pet?: Adopters and pet selection preferences in five animal shelters in the United States. Animals (Basel) 2: 144-159. [Medline] [CrossRef] 